\title{
Challenges in using Compliant Ligaments for Position Estimation within Robotic Joints
}

\author{
Felix Russell ${ }^{1}$, Lei $\mathrm{Gao}^{2}$, Peter Ellison ${ }^{1}$ and Ravi Vaidyanathan ${ }^{1}$
}

\begin{abstract}
The mechanical advantages of bio-inspired condylar robotic knee joints for use in prosthetics or rehabilitation has been argued extensively in literature. A common limitation of these designs is the difficulty of estimating joint angle and therefore accurately controlling the joint. Furthermore, the potential role of ligament-like structures in robotic knees is not very well established. In this work, we investigate the role of compliant stretch sensing ligaments and their integration into a condylar robotic knee. Simulations and experiments are executed out in order to establish whether measurement of stretch in these structures can be used to produce a new feedback controller for joint position. We report results from a computer model, as well as the design and construction of a robotic knee that show, for a chosen condyle shape, ligament stretch is a function of muscle force and joint velocity as well as joint angle. We have developed a genetic algorithm optimised controller incorporating ligament feedback that demonstrates improved performance for a desired joint angle in response to step inputs. The controller showed marginal improvement in response to a cyclic command signal and further investigation is required in order to use these measurements in robust control, nevertheless we believe these results demonstrate the that ligament-like structures have the potential to improve the performance of robotic knees for prosthetics and rehabilitation devices.
\end{abstract}

\section{INTRODUCTION}

Biomimetic robots take inspiration from structures and systems seen in nature in order to produce improved performance. In the field of robotics there is currently interest in developing walking robots that harness natural limb dynamics in order to reduce energy consumption [1], [2]. This has been done by adding elastic elements, such as torsional springs, to the joint system. It has also been shown that the mechanical structure of the human knee, two condyles connected with ligaments, has some mechanical advantages over a simple pin joint [3]. There is, however, no current robust method for estimating the angle of such a joint for use in a controller since the polycentric nature of the joint makes the incorporation of potentiometers or encoders into the mechanism challenging. A potential solution to this problem is to build stretch sensing elastic ligaments into the robotic knee. It could then be possible to estimate the angle of the condylar joint by measuring the strain in these ligaments and this information could be used in an improved controller for the joint. At the same time, incorporating

\footnotetext{
${ }^{1}$ Felix Russell, Peter Ellison and Ravi Vaidyanathan are with the Department of Mechanical Engineering, Imperial College London, UK felix.russell12@ic.ac.uk, p.ellisoneic.ac.uk, r.vaidyanathan@ic.ac.uk

${ }^{2}$ Lei Gao is with the College of Mechanical Engineering, Zhejiang University, China gaolei@zju.edu.cn
}
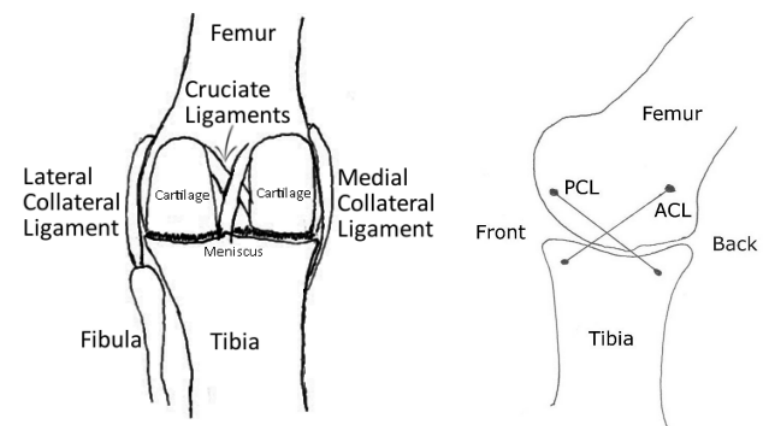

Fig. 1. Layout of the human knee joint. The left hand figure shows the view from behind the joint and the right hand figure shows the view from the inside looking outwards

these elastic elements may also provide some ability to store energy during gait, enhancing limb dynamics. The new joint could be used for prosthetics or rehabilitation devices in order provide improved functionality over currently available mechanisms.

\section{A. Physiology of the human knee}

The human knee is a synovial joint, the largest in the human body. A layer of smooth cartilage covers the femoral and tibial condyles which allows low friction sliding between the joint surfaces. The conydles make contact throughout joint motion, allowing the knee to take compressive loads that, during activities such as stair descent, are $>2.5 \times$ BodyWeight [4]. The two condyles are fixed together by soft tissues which include four ligaments that act to resist movement of the tibia outside of the normal range [5]. These ligaments are compliant, having very low stiffness under small strains and linearly elastic properties under higher strains up to the point of failure [6]. The four ligaments are the anterior crutiate ligament (ACL) and posterior crutiate ligament (PCL) - these are found in the centre of the knee (Figure 1) - and the medial and lateral collateral ligaments (MCL and LCL) which are found on the sides of the joint.

The human knee is tendon driven with the hamstrings and quadriceps being the primary muscle groups attached to the tibia to provide actuation in the flexion and extension directions. During motion both muscles provide a force in the contracting direction and the joint accelerates in the direction of the stronger torque. The agonist is the one which contracts and the antagonist extends. During slow isokinetic contraction it has been found that the antagonist exerts a near constant resistive torque on the joint and it is theorised that this aids the ligaments in maintaining joint stability [7]. 
The patellar sits on the front of the joint and connects the tibia and the quadriceps via the patella tendon. It provides a mechanical advantage to the quadriceps and applies a reaction force onto the femoral condyle, pulling the femoral condyle backwards relative to the tibial condyle [8]. It therefore plays an important roll in the system of forces within the knee mechanism.

There is evidence from microscope observations that there are mechanoreceptors present within the ligaments [9]. Discharge from these receptors has been measured [10] and it is now thought likely that these signals are used to modify muscle stiffness [11]. It has generally been agreed that the afferent signals controlling joint stability interact in a complex manner within the wider sensorimotor system [12] there is, however, no doubt that they play an important roll in joint control. This is evidenced by tests in cats and humans that have shown that removing or deafferenting these structures can impair joint function [13], [14], [15]. For a full review on the sensory role of crutiate ligaments see Johansson et al [16]. There may therefore be some benefit in incorporating the physical and neurosensory features of the human knee into a robotic knee.

The aim of this work is to explore the possibility of using compliant ligament-like structures with stretch sensing capability in a robotic knee with a view to later implementing the measurement of stretch in an improved controller for walking robots. To do this an over-actuated robotic model of the knee is developed which includes ligament analogues which contain sensors to measure their stretch. This will allow the evaluation of the extent to which different factors such as actuator force, direction of joint motion and amount of antagonistic force affect how the ligaments stretch during motion and so the feasibility of developing an improved controller. The robotic knee will be condylar and tendon driven with actuators for the hamstrings and quadriceps acting as an antagonistic pair. The robot will be complemented by a Simscape Multibody model of the same system. The implementation and proper investigation of the control strategy will be the subject of future work.

\section{B. Related work}

Polycentric knees have been used in a number of walking robots and prosthetics because they can improve joint performance by improving toe clearance during swing phase [17] or to improve a walking robot's torque-angle curve, removing the low mechanical advantage regions that can occur at the extremes of motion in a pin jointed mechanism [18]. A condylar hinge is an example of a polycentric mechanism. A design for such a mechanism for use in walking robots and prosthetics has already been developed by Etoundi et al [3]. This joint has no sensing capability within the ligaments but demonstrates the advantages of the mechanism when compared to a pin joint with both lower peak muscle forces (for a given torque) and an ability to take large compressive loads. An alternative condylar hinge design by Jeanneau et al [19] uses a flexible material attached to both condyles to

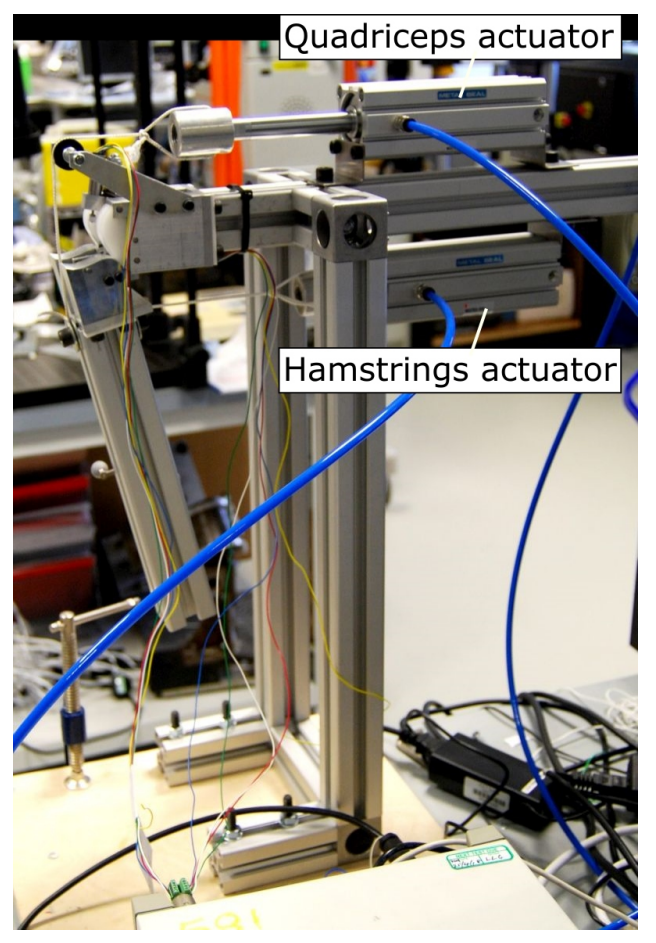

Fig. 2. Overview of the physical simulation

insure pure rolling contact. This hinge has already been put to use in the passive-dynamic walking robot LARP [20].

\section{METHODS}

The design of the prototype knee is shown in Figure 2. The human knee requires collateral ligaments on the sides of the joint in order to stop abduction and to resist tibial torsion. The mechanical joint has been designed such that condyles have constant cross-section in sagital plane, this simplification is designed to make the mechanism more stable and removes the need for these ligaments. The ACL and PCL will be the only ligaments included in the model.

Low friction pneumatic actuators have been selected to simulate the hamstrings and quadriceps. The actuator force is changed by adjusting the cylinder pressure using an electronic pressure controller. A pulley is used to simulate the patellar and nylon cord has been used to simulate the hamstring and patellar tendons.

\section{A. Ligament design}

Figure 3 shows the designs for the compliant ligamentlike structures used within the robotic knee. The two pivots can move apart subject to force from the springs. Like the human ligaments the mechanical versions can only apply force in tension. The spring stiffness used is $126 \mathrm{~N} / \mathrm{mm}$, a value that closely matched those recorded by Jones et al [6]. In parallel with the spring is a linear variable differential transformer (LVDT) with it's coil and core attached to the two pieces of the compliant ligament. This provides the means for the precise measurement of ligament stretch in real time. The unstretched length of the ligaments can be adjusted by tightening a nut, $35 \mathrm{~mm}$ was used in the simulations 


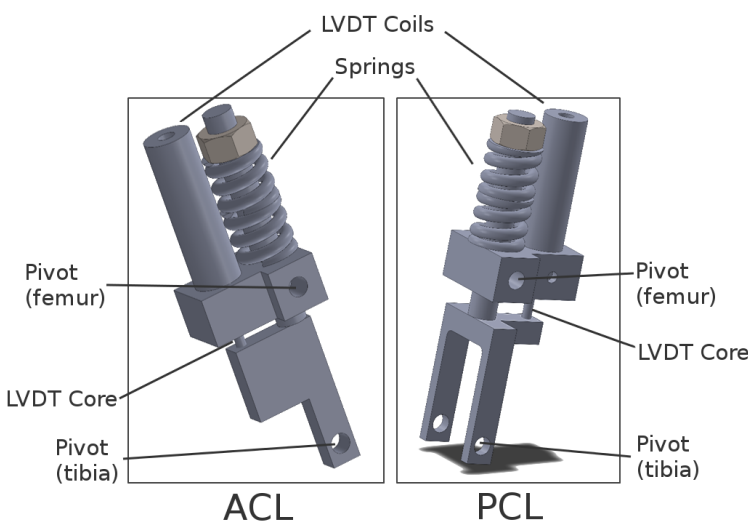

Fig. 3. Ligament design

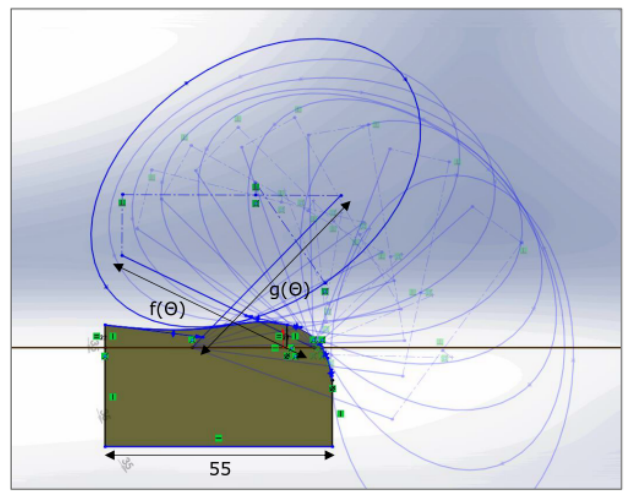

Fig. 4. Method for the design of the tibial condyle detailed in this paper to match measurements by Cohen et al [21].

\section{B. Condyle design}

A low friction polymer, acetal, was chosen for the femoral condyle to enable sliding between the two surfaces. Figure 5 shows a detailed view of the joint. The profile of the tibial condyle was selected in order to generate a particular amounts of ligament stretch at each angle of rotation. Kurosawa et al [22] measured the strain in the ligaments at different angles of the knee and found that within each bundle there was variation in the amount of strain. For the purposes of this model ligaments lengths were selected so that the ACL and PCL lengths varied as a function of joint angle.

Having selected a shape for the femoral condyle and attachment points for the ligaments, the joint was rotated, adjusting the ligament lengths to preselected values as the angle changed (Figure 4). At each rotation the tibial profile was adjusted in order to be in contact with the femoral condyle at a single point without interfering at any point. This is a similar method to that employed by Etoundi et al [3].

\section{Measurement and control}

Figure 6 shows a schematic of the whole system. An infrared tracking camera, Atracsys Fusiontrack 500, was used to

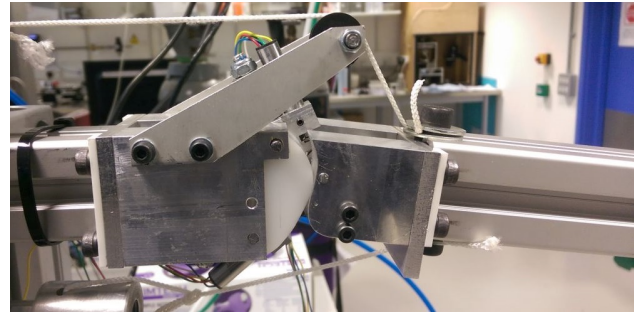

Fig. 5. Detailed view of the assembled joint measure the joint position.

\section{Computer model}

A Simulink computer model of the same system was developed alongside the physical model. the contact model used required that the condyle geometry be simplified to a series of constant radius arcs. Hertz contact theory was used to model the interaction between the acetal femoral condyle and the aluminium tibial condyle. Within the simulation environment it was possible to precisely measure the performance of any given controller using a criterion proposed by Gaing [23]

$$
W(K)=\left(1-e^{-\beta}\right) \cdot\left(M_{p}+E_{s s}\right)+e^{-\beta} \cdot\left(t_{s}-t_{r}\right)
$$

Where $\beta$ is a weighting factor which was set to 0.1 for these simulations, $M_{p}$ the overshoot, $E_{s s}$ the steady state error, $t_{s}$ the settling time and $t_{r}$ the rise time. PID control parameters were then optimised with a genetic algorithm in order to minimise this performance parameter.

\section{RESULTS}

Simulations on the robot were run multiple times to test the repeatability of the measurements. A test run 6 times with the same settings produced a near identical ligamentjoint angle curve each time.

A number of simple simulations were run on the system in order to show how different loads from the muscles affected the way the compliant ligaments stretched during joint motion. Figure 7 shows the result of a test in which the leg, held initially in flexion by a small force from the hamstring actuator, was subjected to a step change in force that caused the knee to extend. The test was repeated under different antagonistic loads from the hamstrings. It can be seen that increasing the hamstrings force decreases the amount of ligament stretch when the joint angle is less than 40 degrees and increases it when the joint angle is more than 50 degrees. For the PCL a decrease is seen at the two extremes of motion and an increase is seen between 60 and 80 degrees.

Tests were also completed whereby the amount of hamstring force was kept at a low level and a step change of only the quadriceps actuator force was applied (Figure 8). Again the amount of ligament stretch changed depending on the loading conditions. It was found that ligament stretch was higher for both ligaments with higher force when the joint angle was small (less than 40 degrees). For the PCL stretch decreased with larger force for $\theta<60^{\circ}$. For the ACL stretch decreased for $55^{\circ}<\theta<90^{\circ}$ and increased for $\theta>90^{\circ}$. 


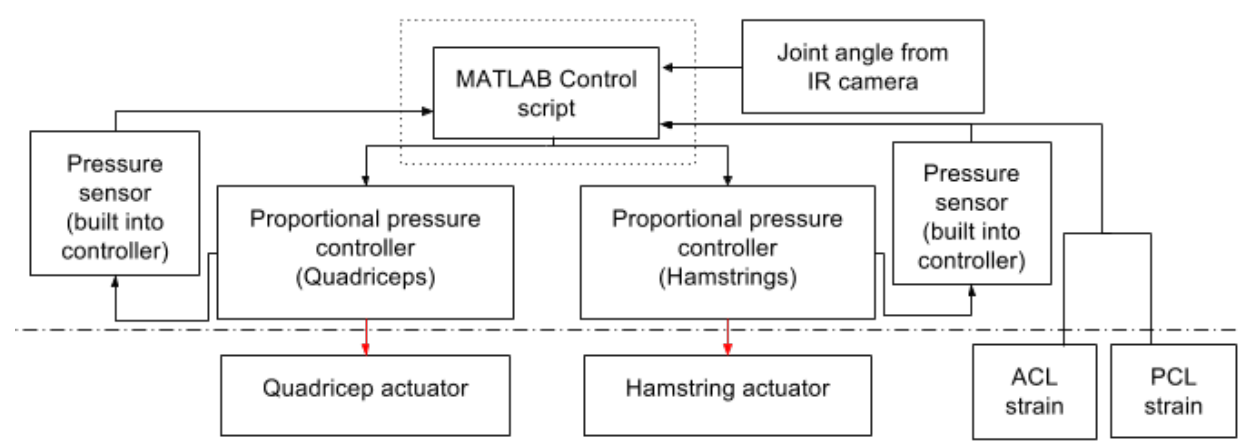

Fig. 6. Schematic of the simulator
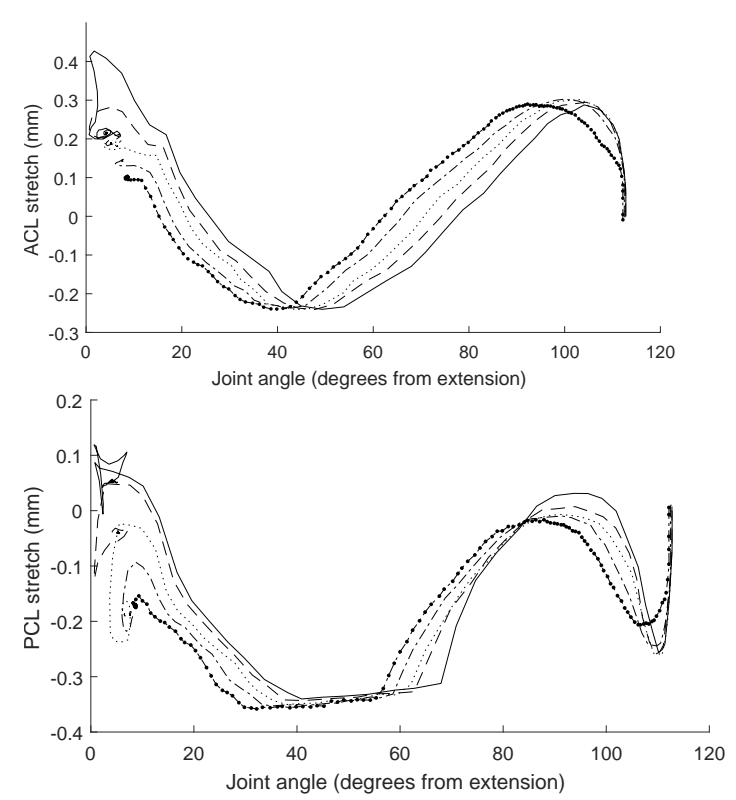

\begin{tabular}{ccc}
\hline Linestyle & Quad's actuator force & Hamstr's actuator force \\
\hline & $50 \mathrm{~N}$ & $0 \mathrm{~N}$ \\
\hline----- & $60 \mathrm{~N}$ & $10 \mathrm{~N}$ \\
\hline & $70 \mathrm{~N}$ & $20 \mathrm{~N}$ \\
\hline$\ldots \ldots+$ & $80 \mathrm{~N}$ & $30 \mathrm{~N}$ \\
\hline$\ldots \ldots \ldots$ & $90 \mathrm{~N}$ & $40 \mathrm{~N}$ \\
\hline
\end{tabular}

Fig. 7. Effect of increasing the antagonistic muscle force on ACL and PCL stretch. Starting in flexion the actuator forces are increased stepwise to the values in the table above from $0 \mathrm{~N}$

Cyclic tests were also run on the robotic model. This was done using a sinusoidal command signal passed to a Zeigler-Nichols tuned PID controller. Figure 9 shows ligament stretch for the ACL and PCL as this cyclic movement takes place. It can be seen that ligament stretch is different depending on the direction of travel of the knee joint.

In the small region $32^{\circ}<\theta<38^{\circ}$ of the curve seen in Figure 9 the relationship between ACL stretch and joint angle appears to act reasonably independently of joint velocity. As a proof of concept a controller was implemented that used the ACL ligament stretch to estimate joint angle and then used that information to achieve a particular target position. The system worked successfully within that range, with a steady state error of $1.2^{\circ}$ compared to $0.5^{\circ}$ when using direct feedback from the tracking camera. The performance
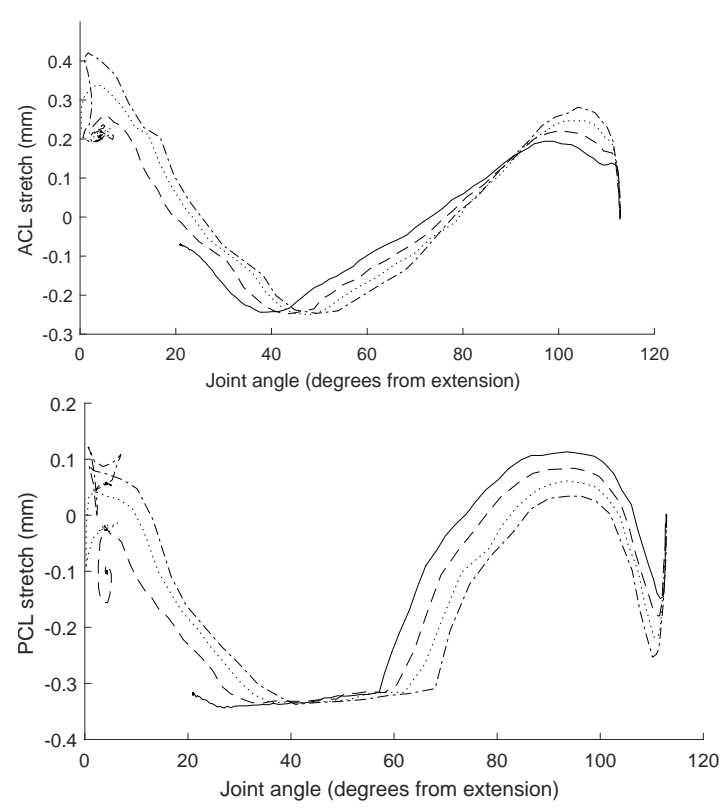

\begin{tabular}{ccc}
\hline Linestyle & Quad's actuator force & Hamstr's actuator force \\
\hline- & $20 \mathrm{~N}$ & $0 \mathrm{~N}$ \\
\hline------ & $30 \mathrm{~N}$ & $0 \mathrm{~N}$ \\
\hline$\cdots \cdots \cdots \cdots$ & $40 \mathrm{~N}$ & $0 \mathrm{~N}$ \\
\hline$-\cdot \cdot-\cdot \cdot-\cdot$ & $50 \mathrm{~N}$ & $0 \mathrm{~N}$ \\
\hline
\end{tabular}

Fig. 8. Effect of different magnitudes of stepwise increase in quadriceps force when the joint started at maximum flexion with an initial quadriceps force of $0 \mathrm{~N}$. The hamstring actuator was set to $0 \mathrm{~N}$ throughout.

criterion in Equation 1 was deemed unsuitable for use on the physical model because of sensitivity to experimental variability - small amounts of unpredictable stiction between the condyles could dramatically change the value of $W(K)$. With the current model it is not possible to predict the joint angle using ligament feedback alone outside of that small range, at this stage we sought only to prove the possibility of using ligament feedback for joint control. Refinement of the technique is a matter for future work.

In the computer model the optimal PID paramaters were found using the performance paramaters and method discussed in section II-D. An alternative controller (Figure 10) was developed that included an additional PID controller which used ACL stretch. The six parameters of the two controllers were again GA optimised for $W(K)$. For a step 

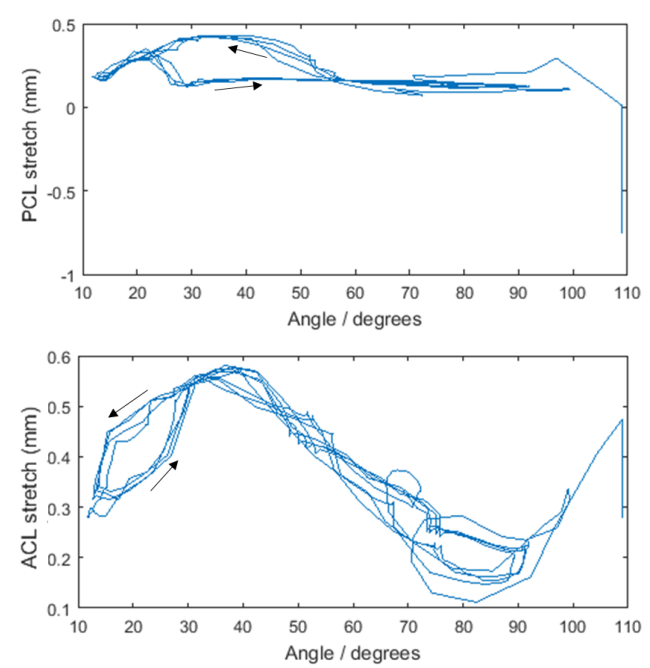

Fig. 9. Effect of moving in different directions on ligament stretch

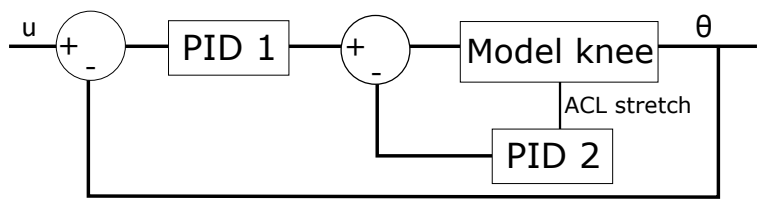

Fig. 10. Control schematic for the computer model using ligament feedback from the ACL to achieve command angle $u$

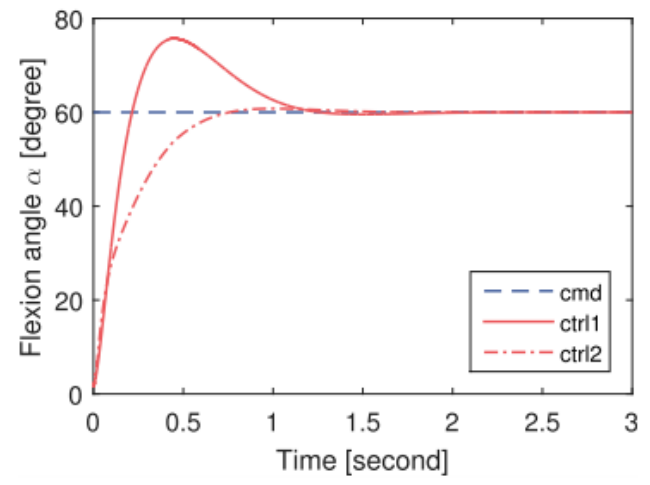

Fig. 11. Comparison of the two control stratagies for step input: ctrll=with only angle feedback. ctrl2=with angle and ACL stretch feedback

change in command signal it was found that the controller that used joint angle and ligament stretch had a 22-52.8\% lower $W(K)$ compared to the one that used only joint angle (Figure 11 and Table I). When tested on a cyclic command signal very little improvement was observed. The computer model also showed that ligament stretch was sensitive to muscle loads with the amount that the ligaments stretched changing depending on the magnitude of the overall forces, the direction of motion and the amount of antagonistic force from the muscle opposing the motion.

\section{DISCUSSION}

The results in Figure 7 can be explained by looking at the forces on the robotic knee. Near full extension in Figure 12 the hamstrings force acts to pull the joint downwards, allowing the tibial condyle to move to the right, reducing ligaments stretch. For $55^{\circ}<\theta<80^{\circ}$, stretch in both
TABLE I

PERFORMANCE IMPROVEMENT WHEN LIGAMENT FEEDBACK IS INCORPORATED INTO THE SIMULINK MODEL OF THE JOINT

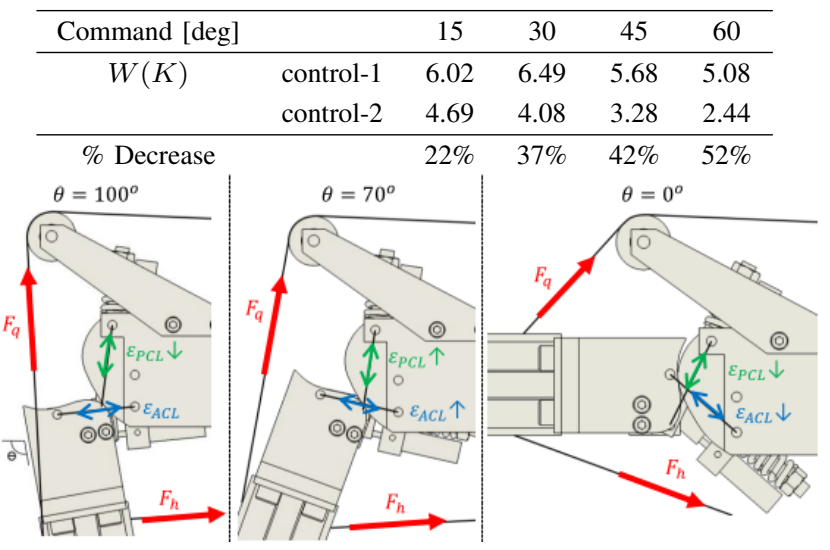

Fig. 12. Diagram explaining the change in ligament stretch. The arrows show whether the ligament stretch increases or decreases as both hamstring and quadriceps actuator forces are increased as per the legend in Figure 7

ligaments is increased. This could be because the hamstring force induces slip and so the tibia and femur are pulled further apart by the condyle shape. At full flexion the reduction in PCL stretch could be because the increased quadriceps force is acting at such an angle that its effect is greater than the force from the hamstrings, causing the tibial condyle to slip upwards. The PID controller used in the physical model is implemented by varying the net force from both actuators combined. Thus it is hypothesised that in the region $32^{\circ}<\theta<38^{\circ}$ the effects observed in figures 7 and 8 combine and cancel out to give a region in which ACL stretch is independent of the controller net force output. Within the robotic joint force and velocity are correlated and so we see that it is in this region that it is possible to perform early tests showing the use of ligament based control.

The results from both models show that actuator forces and the system dynamics have an effect on the way the ligaments in the model stretched. Studies in cadavers have also shown that muscle forces change ligament strain in human knees [24], [25], [26]. In particular the static test of Draganich et al [26] show that near full extension $\left(\theta=0^{\circ}\right)$ antagonistic force reduces ACL strain which matches the mechanical model data in Figure 7. Many current knee investigation systems make simplifications that do not account for changes in joint behaviour that may occur when moving at speed or under different muscle loads. For example, the Oxford rig [27] allows cadaver joints to be loaded by a single actuator to simulate the quadriceps but does not allow dynamic testing. A system by Fujie et al [28] moves a cadaver slowly using a robot arm, taking measurements throughout. The results here show that these simplifications could significantly change the forces acting within the joint.

The improvements in control strategy observed in the computer model with the addition of ligament feedback are likely due to the added information about joint forces that this extra control loop provides. The results are promising 
and it is hoped that they can be replicated in the physical model in due course. Future work will include a closed chain robotic model that can be subjected to more lifelike loads. It is on this model that the authors hope to show improved control using ligament feedback. The lack of improvement when subjected to a cyclic command signal demonstrates some limitations of the current computer model. Further development in the new robotic model will be carried out in order to ascertain the magnitude of the potential benefits of this type of control strategy.

\section{CONCLUSION}

The results of the robotic simulation highlight the challenges that must be overcome in order to use ligament-like structures within the joint to improve the control of robotic knees. They showed that ligament stretch is a function of not just joint angle but also actuator force and joint velocity and that therefore further investigation is required in order to be able to successfully utilise these compliant features for control. Initial results in a small working range on the robotic model suggest that there could be potential in this area and this is partly supported by results from the computer model that show an improvement in performance when ligament stretch is used in the knee controller for a step input. The potential benefits of a compliant ligament inclusive robotic knee could not be fully assessed in the experiments described here. Future work may include a new closed chain robotic test rig or computer model in order to better quantify control and stability improvements. However, this work has shown that robotic knees for rehabilitation, prosthetics and walking robots could potentially benefit from this compliant ligament technology. This would allow the condylar joint design (with it's various mechanical advantages) to be incorporated into systems that are currently pin jointed while at the same time providing the same standard or better joint control.

\section{REFERENCES}

[1] P. A. Bhounsule, J. Cortell, A. Grewal, B. Hendriksen, J. D. Karssen, C. Paul, and A. Ruina, "Low-bandwidth reflex-based control for lower power walking: $65 \mathrm{~km}$ on a single battery charge," The International Journal of Robotics Research, vol. 33, no. 10, pp. 1305-1321, 2014.

[2] S. H. Collins, M. Wisse, and A. Ruina, "A three-dimensional passivedynamic walking robot with two legs and knees," The International Journal of Robotics Research, vol. 20, no. 7, pp. 607-615, 2001.

[3] A. Etoundi, S. C. Burgess, and R. Vaidyanathan, "A bio-inspired condylar hinge for robotic limbs," ASME Journal of Mechanisms and Robotics, vol. 5, no. 3, pp. 1-8, 2013.

[4] A. Mundermann, C. O. Dyrby, D. D. D'Lima, C. W. Colwell, and T. P. Andriacchi, "In vivo knee loading characteristics during activities of daily living as measured by an instrumented total knee replacement," Journal of Orthopaedic Research, vol. 26, no. 9, pp. 1167-1172, 2008.

[5] F. G. Girgis, J. L. Marshall, and A. A. M. JEM, "The cruciate ligaments of the knee joint: Anatomical. functional and experimental analysis." Clinical orthopaedics and related research, vol. 106, pp. 216-231, 1975.

[6] R. Jones, N. Nawana, M. Pearcy, D. Learmonth, D. Bickerstaff, J. Costi, and R. Paterson, "Mechanical properties of the human anterior cruciate ligament," Clinical Biomechanics, vol. 10, no. 7, pp. 339-344, 1995.

[7] R. Baratta, M. Solomonow, B. Zhou, D. Letson, R. Chuinard, and R. D'ambrosia, "Muscular coactivation: the role of the antagonist musculature in maintaining knee stability," The American journal of sports medicine, vol. 16, no. 2, pp. 113-122, 1988.
[8] V. C. Mow and R. Huiskes, Basic orthopaedic biomechanics \& mechano-biology. Lippincott Williams \& Wilkins, 2005.

[9] T. Hogervorst and R. A. Brand, "Current concepts reviewmechanoreceptors in joint function," J Bone Joint Surg Am, vol. 80, no. 9 , pp. $1365-1378,1998$.

[10] W. Ferrell, "The adequacy of stretch receptors in the cat knee joint for signalling joint angle throughout a full range of movement." The Journal of physiology, vol. 299, p. 85, 1980.

[11] A. W. Kim, A. M. Rosen, V. A. Brander, and T. S. Buchanan, "Selective muscle activation following electrical stimulation of the collateral ligaments of the human knee joint," Archives of physical medicine and rehabilitation, vol. 76, no. 8, pp. 750-757, 1995.

[12] B. L. Riemann and S. M. Lephart, "The sensorimotor system, part i: the physiologic basis of functional joint stability," Journal of athletic training, vol. 37, no. 1, p. 71, 2002.

[13] W. Ferrell, R. Baxendale, C. Carnachan, and I. Hart, "The influence of joint afferent discharge on locomotion, proprioception and activity in conscious cats," Brain research, vol. 347, no. 1, pp. 41-48, 1985.

[14] J. P. Corrigan, W. F. Cashman, and M. P. Brady, "Proprioception in the cruciate deficient knee," Bone \& Joint Journal, vol. 74, no. 2, pp. 247-250, 1992.

[15] G. Pap, A. Machner, W. Nebelung, and F. Awiszus, "Detailed analysis of proprioception in normal and acl-deficient knees," Bone \& Joint Journal, vol. 81, no. 5, pp. 764-768, 1999.

[16] H. Johansson, P. Sjölander, and P. Sojka, "A sensory role for the cruciate ligaments." Clinical orthopaedics and related research, vol. 268, pp. 161-178, 1991.

[17] S. A. Gard, D. S. Childress, and J. E. Uellendahl, "The influence of four-bar linkage knees on prosthetic swing-phase floor clearance." JPO: Journal of Prosthetics and Orthotics, vol. 8, no. 2, pp. 34-40, 1996.

[18] H. Khan, R. Featherstone, D. G. Caldwell, and C. Semini, "Bioinspired knee joint mechanism for a hydraulic quadruped robot," in Automation, Robotics and Applications (ICARA), 2015 6th International Conference on. IEEE, 2015, pp. 325-331.

[19] A. Jeanneau, J. Herder, T. Laliberté, and C. Gosselin, "A compliant rolling contact joint and its application in a 3-dof planar parallel mechanism with kinematic analysis," in ASME 2004 International Design Engineering Technical Conferences and Computers and Information in Engineering Conference. American Society of Mechanical Engineers, 2004, pp. 689-698.

[20] G. C. Gini, F. Moro, M. Folgheraiter, and U. Scarfogliero, A biologically founded design and control of a humanoid biped. INTECH Open Access Publisher, 2009.

[21] S. B. Cohen, C. VanBeek, J. S. Starman, D. Armfield, J. J. Irrgang, and F. H. Fu, "Mri measurement of the 2 bundles of the normal anterior cruciate ligament," Orthopedics, vol. 32, no. 9, 2009.

[22] H. Kurosawa, K.-i. Yamakoshi, K. Yasuda, and T. Sasaki, "Simultaneous measurement of changes in length of the cruciate ligaments during knee motion." Clinical orthopaedics and related research, vol. 265, pp. 233-240, 1991.

[23] Z.-L. Gaing, "A particle swarm optimization approach for optimum design of pid controller in avr system," IEEE transactions on energy conversion, vol. 19, no. 2, pp. 384-391, 2004.

[24] G. Li, T. Rudy, M. Sakane, A. Kanamori, C. Ma, and S.-Y. Woo, "The importance of quadriceps and hamstring muscle loading on knee kinematics and in-situ forces in the acl," Journal of biomechanics, vol. 32, no. 4, pp. 395-400, 1999.

[25] T. J. Withrow, L. J. Huston, E. M. Wojtys, and J. A. Ashton-Miller, "The relationship between quadriceps muscle force, knee flexion, and anterior cruciate ligament strain in an in vitro simulated jump landing," The American journal of sports medicine, vol. 34, no. 2, pp. 269-274, 2006.

[26] L. Draganich and J. Vahey, "An in vitro study of anterior cruciate ligament strain induced by quadriceps and hamstrings forces," Journal of Orthopaedic Research, vol. 8, no. 1, pp. 57-63, 1990.

[27] A. Zavatsky, "A kinematic-freedom analysis of a flexed-knee-stance testing rig," Journal of biomechanics, vol. 30, no. 3, pp. 277-280, 1997.

[28] H. Fujie, K. Mabuchi, S. Woo, G. Livesay, S. Arai, and Y. Tsukamoto, "The use of robotics technology to study human joint kinematics: a new methodology," Journal of biomechanical engineering, vol. 115, no. 3, pp. 211-217, August 1993. [Online]. Available: http://dx.doi.org/10.1115/1.2895477 